ALEA, Lat. Am. J. Probab. Math. Stat. 13, 925-940 (2016)

DOI: 10.30757/ALEA.v13-35

\title{
Weak Concentration for First Passage Percolation Times on Graphs and General Increasing Set-valued Processes
}

\author{
David J. Aldous \\ Statistics Department \\ 367 Evans Hall \# 3860, \\ U.C. Berkeley CA 94720, USA. \\ E-mail address: aldous@stat.berkeley.edu \\ $U R L$ : www.stat.berkeley.edu/users/aldous
}

\begin{abstract}
A simple lemma bounds s.d.( $T) / \mathbb{E} T$ for hitting times $T$ in Markov chains with a certain strong monotonicity property. We show how this lemma may be applied to several increasing set-valued processes. Our main result concerns a model of first passage percolation on a finite graph, where the traversal times of edges are independent Exponentials with arbitrary rates. Consider the percolation time $X$ between two arbitrary vertices. We prove that s.d. $(X) / \mathbb{E} X$ is small if and only if $\Xi / \mathbb{E} X$ is small, where $\Xi$ is the maximal edge-traversal time in the percolation path attaining $X$.
\end{abstract}

\section{Introduction}

For random variables $T$ arising (loosely speaking) as optimal costs in some "random environment" model, one might not be able to estimate $\mathbb{E} T$ explicitly, for one of two reasons: it may involve a difficult optimization problem (exemplified by the Euclidean TSP over random points (Steele, 1997), or the model may involve many parameters (the case more relevant to this paper). In such cases the well-known method of bounded differences (McDiarmid, 1989) often enables us to bound $T-\mathbb{E} T$ explicitly, and this general topic of concentration inequalities has been developed in many directions over the last generation. What we will call (recalling the weak law of large numbers) a weak concentration inequality is just a result showing s.d.(T)/ET is small. The starting point for this paper is a simple technique (section 1.1) for proving weak concentration for hitting times $T$ in Markov chains with a certain

Received by the editors June 16, 2015; accepted on September 15, 2015 and in its final version on September 21, 2016.

2010 Mathematics Subject Classification. 60J27, 60K35, 05C82.

Key words and phrases. Concentration inequality, monotone, Markov chain, coupling, first passage percolation.

Research supported by N.S.F Grant DMS-1504802. 
strong monotonicity property. At first sight one might doubt that this technique could be more than very narrowly applicable, but its quick use in diverse other contexts (sections 1.2 - 1.4; see also section $5(\mathrm{~d})$ ) should partly dispel such doubts. Our main result concerns a more sophisticated analysis of a model of first passage percolation on a finite graph, where the traversal times of edges are independent Exponentials with arbitrary rates. We will describe that result and its background in section 2 , and prove it in the subsequent sections.

1.1. A monotonicity condition. The setting is a continuous-time Markov chain $\left(Z_{t}\right)$ on a finite state space $\Sigma$, where we study the hitting time

$$
T:=\inf \left\{t: Z_{t} \in \Sigma_{0}\right\}
$$

for a fixed subset $\Sigma_{0} \subset \Sigma$. Assume

$$
h(S):=\mathbb{E}_{S} T<\infty \text { for each } S \in \Sigma
$$

which holds under the natural "reachability" condition - that is, if it is possible to reach $\Sigma_{0}$ eventually from each initial state. Assume also a rather strong "monotonicity" condition:

$$
h\left(S^{\prime}\right) \leq h(S) \text { whenever } S \rightarrow S^{\prime} \text { is a possible transition. }
$$

In a typical example, the state space $\Sigma$ will be the space of all subsets $S$ of a given finite set $\mathbf{V}$, and possible transitions will be of the form $S \rightarrow S \cup\{v\}$. A special case of our simple lemma, which suffices for the quick applications in sections 1.2 1.4 , is

Lemma 1.1. Under condition (1.3), for any initial state,

$$
\frac{\operatorname{var} T}{\mathbb{E} T} \leq \max \left\{h(S)-h\left(S^{\prime}\right): S \rightarrow S^{\prime} \text { a possible transition }\right\} .
$$

In the "quick applications" below, the right side will be bounded using natural couplings of the processes started from $S$ and from $S^{\prime}$. For more complicated applications, Lemma 1.2 allows the possibility of occasional transitions for which $h(S)-h\left(S^{\prime}\right)$ is not so small.

Lemma 1.2. In the setting of Lemma 1.1, for arbitrary $\delta, \varepsilon>0$,

$$
\frac{\operatorname{var} T}{(\mathbb{E} T)^{2}} \leq \delta+\varepsilon+\frac{\mathbb{E} \int_{0}^{T} \mathbb{1}_{\left\{q_{\delta}\left(Z_{u}\right) \geq \varepsilon\right\}} d u}{\mathbb{E} T} .
$$

where $q_{\delta}(S)$ is defined at (1.9) below.

The proofs below involve only very standard martingale analysis. We are not claiming any conceptual novelty in these results, but instead emphasize their applications later. A variant form of Lemma 1.2 is derived in Aldous (2016) Corollary 2.3.

Proof of Lemma 1.1: Expectation is relative to some fixed initial state $S_{0}$. Note that $T=\inf \left\{t: h\left(Z_{t}\right)=0\right\}$, that $t \rightarrow h\left(Z_{t}\right)$ is decreasing (by which we always mean non-increasing), by (1.3), and so for any reachable state $S$ we have $h(S) \leq$ $h\left(S_{0}\right)=\mathbb{E} T$, facts we use frequently without comment. Consider the martingale

$$
M_{t}:=\mathbb{E}\left(T \mid Z_{t}\right)=h\left(Z_{t \wedge T}\right)+t \wedge T .
$$


The Doob-Meyer decomposition of $M_{t}^{2}$ into a martingale $Q_{t}$ and a predictable process is clearly

$$
M_{t}^{2}-M_{0}^{2}=Q_{t}+\int_{0}^{t} a\left(Z_{u}\right) d u
$$

where

$$
a(S):=\sum_{S^{\prime}} q\left(S, S^{\prime}\right)\left(h(S)-h\left(S^{\prime}\right)\right)^{2}
$$

where $q\left(S, S^{\prime}\right)$ are the transition rates. Taking expectation at $t=\infty$ gives

$$
\operatorname{var} T=\mathbb{E} \int_{0}^{T} a\left(Z_{u}\right) d u
$$

On the other hand the martingale property (1.4) for $\mathbb{E}\left(T \mid Z_{t}\right)$ corresponds to the identity

$$
b(S):=\sum_{S^{\prime}} q\left(S, S^{\prime}\right)\left(h(S)-h\left(S^{\prime}\right)\right)=1 \text { while } S \cap \Sigma_{0}=\emptyset
$$

and therefore

$$
\mathbb{E} T=\mathbb{E} \int_{0}^{T} b\left(Z_{u}\right) d u
$$

Setting

$$
\kappa=\max \left\{h(S)-h\left(S^{\prime}\right): S \rightarrow S^{\prime} \text { a possible transition }\right\}
$$

we clearly have $a(S) \leq \kappa b(S)$; note this is where we use the monotonicity hypothesis (1.3). The result now follows from $(1.6,1.8)$.

Proof of Lemma 1.2: We continue with the notation above. Fix $\delta>0$ and write, for a possible transition $S \rightarrow S^{\prime}$,

$$
h(S)-h\left(S^{\prime}\right) \leq \delta \mathbb{E} T+\mathbb{1}_{\left\{h(S)-h\left(S^{\prime}\right)>\delta \mathbb{E} T\right\}} \mathbb{E} T .
$$

Using this to bound one term of the product $\left(h(S)-h\left(S^{\prime}\right)\right)^{2}$ in the definition (1.5) of $a(S)$, and comparing with the definition (1.7) of $b(S)$, we obtain

$$
\begin{aligned}
a(S) \leq & b(S) \cdot \delta \mathbb{E} T \\
& +\sum_{S^{\prime}: h(S)-h\left(S^{\prime}\right)>\delta \mathbb{E} T} q\left(S, S^{\prime}\right)\left(h(S)-h\left(S^{\prime}\right)\right) \cdot \mathbb{E} T
\end{aligned}
$$

While $S \cap \Sigma_{0}=\emptyset$ we have $b(S)=1$ and so

$$
\frac{a(S)}{\mathbb{E} T} \leq \delta+q_{\delta}(S)
$$

where

$$
q_{\delta}(S):=\sum_{S^{\prime}: h(S)-h\left(S^{\prime}\right)>\delta \mathbb{E} T} q\left(S, S^{\prime}\right)\left(h(S)-h\left(S^{\prime}\right)\right)
$$

Using (1.6)

$$
\frac{\operatorname{var} T}{\mathbb{E} T} \leq \delta \mathbb{E} T+\mathbb{E} \int_{0}^{T} q_{\delta}\left(Z_{u}\right) d u .
$$

Because $q_{\delta}(S) \leq b(S) \equiv 1$ we can fix $\varepsilon>0$ and write $q_{\delta}\left(Z_{u}\right) \leq \varepsilon+\mathbb{1}_{\left\{q_{\delta}\left(Z_{u}\right) \geq \varepsilon\right\}}$, and the result follows. 
Discrete-time chains. Given a discrete-time Markov chain with transition probabilities $p\left(S, S^{\prime}\right)$ there is a corresponding continuous-time Markov chain with transition rates $q\left(S, S^{\prime}\right)=p\left(S, S^{\prime}\right)$. The relation between the hitting times $T_{\text {disc }}$ and $T_{\text {cont }}$ for these two chains is (using Wald's identity)

$$
\mathbb{E} T_{\text {cont }}=\mathbb{E} T_{\text {disc }} ; \quad \text { var } T_{\text {cont }}=\text { var } T_{\text {disc }}+\mathbb{E} T_{\text {disc }} .
$$

Via this continuization device our results may be applied to discrete-time chains.

1.2. A general Markovian growth process. For a straightforward application of Lemma 1.1 , we consider a general growth process $\left(Z_{t}\right)$ on the lattice $\mathbb{Z}^{2}$. The states are finite vertex-sets $S$, the possible transitions are $S \rightarrow S \cup\{v\}$ where $v$ is a vertex adjacent to $S$. For each such transition, we assume the transition rates are bounded above and below:

$$
0<c_{*} \leq q(S, S \cup\{v\}) \leq c^{*}<\infty .
$$

Initially $Z_{0}=\{\mathbf{0}\}$, where $\mathbf{0}$ denotes the origin. The "monotonicity" condition we impose is that these rates are increasing in $S$ :

$$
\text { if } v, v^{\prime} \text { are adjacent to } S \text { then } q(S, S \cup\{v\}) \leq q\left(S \cup\left\{v^{\prime}\right\}, S \cup\left\{v, v^{\prime}\right\}\right) \text {. }
$$

Note that we do not assume any kind of spatial homogeneity.

Proposition 1.3. Let $A$ be an arbitrary non-empty subset of vertices $\mathbb{Z}^{2} \backslash\{\mathbf{0}\}$, and consider $T:=\inf \left\{t: Z_{t} \cap A\right.$ is non-empty. $\}$. Under assumptions (1.12, 1.13),

$$
\operatorname{var} T \leq \mathbb{E} T / c_{*} \text {. }
$$

Proof: First note $\mathbb{E} T<\infty$, because (1.12) implies $\mathbb{E} T \leq \ell / c_{*}$, where $\ell$ is the length of the shortest path from 0 to $A$. Condition (1.13) allows us to couple versions $\left(Z_{t}^{\prime}, Z_{t}^{\prime \prime}\right)$ of the process starting from states $S^{\prime} \subset S^{\prime \prime}$, such that in the coupled process we have $Z_{t}^{\prime} \subseteq Z_{t}^{\prime \prime}$ for all $t \geq 0$. In particular, $h(S):=\mathbb{E}_{S} T$ satisfies condition (1.3). To deduce the result from Lemma 1.1 we need to show that, for any given possible transition $S_{0} \rightarrow S_{0} \cup\left\{v_{0}\right\}$, we have

$$
h\left(S_{0}\right) \leq h\left(S_{0} \cup\left\{v_{0}\right\}\right)+1 / c_{*} .
$$

Now by running the process started at $S_{0}$ until the first time $T^{*}$ this process contains $v_{0}$, and then coupling the future of that process to the process started at $S_{0} \cup\left\{v_{0}\right\}$, we have $h\left(S_{0}\right) \leq \mathbb{E}_{S_{0}} T^{*}+h\left(S_{0} \cup\left\{v_{0}\right\}\right)$. And $\mathbb{E}_{S_{0}} T^{*} \leq 1 / c_{*}$ by (1.12), establishing (1.14).

1.3. A multigraph process. The process here arises in a broad "imperfectly observed networks" program described in Aldous and Li (2016). We give two examples of the application of Lemma 1.1 to this process.

Take a finite connected graph $(\mathbf{V}, \mathbf{E})$ with edge-weights $\mathbf{w}=\left(w_{e}\right)$, where $w_{e}>$ $0 \forall e \in \mathbf{E}$. Define a multigraph-valued process as follows. Initially we have the vertex-set $\mathbf{V}$ and no edges. For each vertex-pair $e=(v y) \in \mathbf{E}$, edges $v y$ appear at the times of a Poisson (rate $w_{e}$ ) process, independent over $e \in \mathbf{E}$. So at time $t$ the state of the process, $Z_{t}$ say, is a multigraph with $N_{e}(t) \geq 0$ copies of edge $e$, where $\left(N_{e}(t), e \in \mathbf{E}\right)$ are independent Poisson $\left(t w_{e}\right)$ random variables.

We study how long until $Z_{t}$ has various connectivity properties. Specifically, consider

$$
\text { - } T_{k}^{\text {span }}=\inf \left\{t: Z_{t} \text { contains } k \text { edge-disjoint spanning trees }\right\} \text {. }
$$


- $T_{k}^{\text {tria }}=\inf \left\{t: Z_{t}\right.$ contains $k$ edge-disjoint triangles $\}$.

Here we regard the $N_{e}(t)$ copies of $e$ as disjoint edges. Remarkably, Lemma 1.1 enables us to give simple proofs of "weak concentration" bounds which do not depend on the underlying weighted graph.

\section{Proposition 1.4.}

$$
\frac{\text { s.d. }\left(T_{k}^{\text {span }}\right)}{\mathbb{E} T_{k}^{\text {span }}} \leq \frac{1}{\sqrt{k}}, k \geq 1 .
$$

If $\mathbf{E}$ contains at least one triangle then

$$
\frac{\text { s.d. }\left(T_{k}^{\text {tria }}\right)}{\mathbb{E} T_{k}^{\text {tria }}} \leq\left(\frac{e}{e-1}\right)^{1 / 2} k^{-1 / 6}, k \geq 1 .
$$

Using the continuization device at (1.11) and Wald's identity, the same bounds (in fact, slightly better bounds) hold in the discrete-time model where edges $e$ arrive IID with probabilities proportional to $w_{e}$.

We conjecture that some similar result holds for

$$
T_{k}^{\prime}:=\inf \left\{t: Z_{t} \text { is } k \text {-edge-connected }\right\} .
$$

But proving this by our methods would require some structure theory (beyond Menger's theorem) for $k$-edge-connected graphs, and it is not clear whether relevant theory is known.

Proof of (1.15): First note that because $(\mathbf{V}, \mathbf{E})$ is finite and connected, it contains a spanning tree with minimum edge-weight $w_{*}>0$, implying $\mathbb{E} T_{1}^{\text {span }} \leq(|\mathbf{V}|-$ 1) $/ w_{*}<\infty$ and then $\mathbb{E} T_{k}^{\text {span }} \leq k \mathbb{E} T_{1}^{\text {span }}<\infty$. (A similar argument shows $\mathbb{E} T_{k}^{\text {tria }}<$ $\infty$ under the assumption of (1.16).) We will apply Lemma 1.1. Here the states $S$ are multigraphs over $\mathbf{V}$, and $h(S)$ is the expectation, starting at $S$, of the time until the process contains $k$ edge-disjoint spanning trees. What are the possible values of $h(S)-h(S \cup\{e\})$, where $S \cup\{e\}$ denotes the result of adding an extra copy of $e$ to the multigraph $S$ ?

Consider the "min-cut" over proper subsets $S \subset \mathbf{V}$

$$
\gamma:=\min _{S} w\left(S, S^{c}\right)
$$

where $w\left(S, S^{c}\right)=\sum_{v \in S, y \in S^{c}} w_{v y}$. Because a spanning tree must have at least one edge across the min-cut,

$$
\mathbb{E} T_{k}^{\text {span }} \geq k / \gamma
$$

On the other hand we claim

$$
h(S)-h(S \cup\{e\}) \leq 1 / \gamma .
$$

To prove this, take the natural coupling $\left(Z_{t}, Z_{t}^{+}\right)$of the processes started from $S$ and from $S \cup\{e\}$, and run the coupled process until $Z_{t}^{+}$contains $k$ edge-disjoint spanning trees. At this time, the process $Z_{t}$ either contains $k$ edge-disjoint spanning trees, or else contains $k-1$ spanning trees plus two trees (regard as edge-sets $\mathbf{t}_{1}$ and $\mathbf{t}_{2}$ ) such that $\mathbf{t}_{1} \cup \mathbf{t}_{2} \cup\{e\}$ is a spanning tree. So the extra time we need to run $\left(Z_{t}\right)$ is at most the time until some arriving edge links $\mathbf{t}_{1}$ and $\mathbf{t}_{2}$, which has mean at most $1 / \gamma$. This establishes (1.18), and then Lemma 1.1 establishes (1.15).

Proof of (1.16): The key ingredient is a coupling construction which will establish 
Lemma 1.5.

$$
\frac{\mathbb{E} T_{1}^{\text {tria }}}{\mathbb{E} T_{k}^{\text {tria }}} \leq a(k):=\inf _{0<q<1} \frac{q}{1-\left(1-q^{3}\right)^{k}} .
$$

Granted Lemma 1.5, we start by repeating the format of the previous proof. To bound $h\left(S^{\prime}\right)-h(S)$ for a possible transition (one added edge) $S \rightarrow S^{\prime}$, we take the natural coupling of the processes started from $S$ and $S^{\prime}$, run until the latter contains $k$ edge-disjoint triangles. At that time the former process contains $k-1$ edge-disjoint triangles, so the mean extra time until it contains $k$ edge-disjoint triangles is bounded (crudely) by $\mathbb{E} T_{1}^{\text {tria }}$. Then Lemma 1.1 says

$$
\frac{\operatorname{var} T_{k}^{\text {tria }}}{\mathbb{E} T_{k}^{\text {tria }}} \leq \mathbb{E} T_{1}^{\text {tria }} .
$$

Combining with Lemma 1.5,

$$
\frac{\text { s.d. }\left(T_{k}^{\text {tria }}\right)}{\mathbb{E} T_{k}^{\text {tria }}} \leq \sqrt{a(k)} .
$$

Because $1-x \leq e^{-x}$ we have $\frac{q}{1-\left(1-q^{3}\right)^{k}} \leq \frac{q}{1-\exp \left(-q^{3} k\right)}$, so taking $q=k^{-1 / 3}$ gives the stated bound.

Proof of Lemma 1.5: Recall $\left(N_{e}(t), 0 \leq t\right)$ is the rate- $w_{e}$ Poisson counting process for occurrence of copies of edge $e$. Consider the following coupling construction, done independently for different $e$. Fix $0<q<1$. Delete edge copies with probability $1-q$, and write $\left(\widetilde{N}_{e}(t), 0 \leq t\right)$ for the process of retained edges; then rescale time by setting $N_{e}^{*}(t)=\widetilde{N}_{e}(t / q)$. So $\left(N_{e}(\cdot), N_{e}^{*}(\cdot)\right)$ is a coupling of two dependent rate- $w_{e}$ Poisson processes. It is enough to show, writing $T$ for $T^{\text {tria }}$,

$$
\frac{\mathbb{E} T_{1}^{*}}{\mathbb{E} T_{k}} \leq \frac{q}{1-\left(1-q^{3}\right)^{k}}
$$

where $T_{1}^{*}$ refers to the $\left(N_{e}^{*}(\cdot), e \in \mathcal{E}\right)$ process. Now $T_{1}^{*} / q$ is the time of appearance of the first triangle in the $\left(\widetilde{N}_{e}(\cdot), e \in \mathcal{E}\right)$ process, and so $T_{1}^{*} / q \leq \widetilde{T}$ where the latter is defined by

$\widetilde{T}=T_{k}$ on the event $A=$ "at least one of the $k$ triangles seen at $T_{k}$ in the $N^{*}(\cdot)$ process is retained in the $\widetilde{N}(\cdot)$ process";

$\widetilde{T}=T_{k}+Y$ otherwise, where $Y$ is the subsequent time until a triangle appears in the $\widetilde{N}(\cdot)$ process, if all edges are deleted at time $T_{k}$.

But $\mathbb{E} Y=\mathbb{E} T_{1} / q=\mathbb{E} T_{1}^{*} / q$, so

$$
\begin{aligned}
\mathbb{E} T_{1}^{*} / q \leq \mathbb{E} \widetilde{T} & =\mathbb{E} T_{k}+\mathbb{P}\left(A^{c}\right) \mathbb{E} Y \\
& \leq \mathbb{E} T_{k}+\left(1-q^{3}\right)^{k} \mathbb{E} T_{1}^{*} / q
\end{aligned}
$$

which rearranges to inequality (1.19).

1.4. Coverage processes. The topic of coverage processes is centered upon spatial or combinatorial variants of the coupon collector's problem; see the monograph Hall (1988) and scattered examples in Aldous (1989). Classical theory concerns lowparameter models for which the cover time $T_{n}$ of a "size $n$ " model can be shown to have a limit distribution after scaling: $\left(T_{n}-a_{n}\right) / b_{n} \rightarrow_{d} \xi$ for explicit $a_{n}, b_{n}$. In many settings, Lemma 1.1 can be used to give a weak concentration result for 
models with much less regular structure. Here is a very simple example, whose one-line proof is left to the reader.

Proposition 1.6. Let $G$ be an arbitrary n-vertex graph, and let $\left(V_{i}, i \geq 1\right)$ be IID uniform random vertices. Let $T$ be the smallest $t$ such that every vertex is contained in, or adjacent to, the set $\left\{V_{i}, 1 \leq i \leq t\right\}$. Then $\operatorname{var} T \leq n \mathbb{E} T$.

For a sequence $\left(G_{n}\right)$ of sparse graphs, $\mathbb{E} T_{n}$ will be of order $n \log n$, so the bound says that s.d. $\left(T_{n}\right) / \mathbb{E} T=O(1 / \sqrt{\log n})$.

\section{The FPP model}

As in section 1.3 we start with a finite connected graph $(\mathbf{V}, \mathbf{E})$ with edge-weights $\mathbf{w}=\left(w_{e}\right)$, where $w_{e}>0 \forall e \in \mathbf{E}$, but the model here is different. To the edges $e \in \mathbf{E}$ attach independent Exponential(rate $w_{e}$ ) random variables $\xi_{e}$. For each pair of vertices $\left(v^{\prime}, v^{\prime \prime}\right)$ there is a random variable $X\left(v^{\prime}, v^{\prime \prime}\right)$ which can be viewed in two equivalent ways:

viewing $\xi_{e}$ as the length of edge $e$, then $X\left(v^{\prime}, v^{\prime \prime}\right)$ is the length of the shortest route from $v$ to $v^{\prime}$;

viewing $\xi_{e}$ as the time to traverse edge $e$, then $X\left(v^{\prime}, v^{\prime \prime}\right)$ is the "first passage percolation" time from $v$ to $v^{\prime}$.

Taking the latter view, we call this the FPP model, and call $X\left(v^{\prime}, v^{\prime \prime}\right)$ the FPP time and $\xi_{e}$ the traversal time. This type of model and many generalizations have been studied extensively in several settings, in particular

- FPP with general IID weights on $\mathbb{Z}^{d}$ (Kesten, 2003; Auffinger et al., 2015)

- FPP on classical random graph (Erdős-Rényi or configuration) models (Bhamidi et al., 2010, 2011)

- and a much broader "epidemics and rumors on complex networks" literature (Draief and Massoulié, 2010; Peng, 2016).

However, this literature invariably starts by assuming some specific graph model; there are very few "general results" which relate properties of the FPP model to properties of a general underlying graph. As an analogy, another structure that can be associated with a weighted finite graph is a finite reversible Markov chain; the established theory surrounding mixing times of Markov chains (Aldous and Fill, 2002; Levin et al., 2009; Montenegro and Tetali, 2006) does contain "general results" relating properties of the chain to properties of the underlying graph.

This article makes a modest start by studying the "weak concentration" property: when it is true that $X\left(v^{\prime}, v^{\prime \prime}\right)$ is close to its expectation? We can reformulate the FPP model as a set-valued process (see section 2.1(a) for details) and then Lemma 1.1 immediately implies the following result.

Proposition 2.1. $\operatorname{var} X\left(v^{\prime}, v^{\prime \prime}\right) \leq \mathbb{E} X\left(v^{\prime}, v^{\prime \prime}\right) / w_{*}$ for $w_{*}:=\min \left\{w_{e}: e \in \mathbf{E}\right\}$.

Bounds of this type are classical on $\mathbb{Z}^{d}$ (Kesten, 1993).

Proposition 2.1 implies that on any unweighted graph $\left(w_{e}=1\right.$ for all edges $\left.e\right)$, the spread of $X=X\left(v^{\prime}, v^{\prime \prime}\right)$ is at most order $\sqrt{\mathbb{E} X}$. For many specific graphs, stronger concentration results are known. For $\mathbb{Z}^{2}$ there is extensive literature (see Chatterjee (2013) for a recent overview) on the longstanding conjecture that the spread is order $(\mathbb{E} X)^{1 / 3}$. For the complete graph (e.g. Aldous, 2013, sec. 7.3) and for sparse random graphs on $n$ vertices (Bhamidi et al., 2010) the spread of $X / \mathbb{E} X$ 
is typically of order $1 / \log n$. For nilpotent Cayley graphs it is known the spread is sublinear (Benjamini and Tessera, 2015).

In contrast, in this paper we study the completely general case where the edgeweights $w_{e}$ may vary widely over the different edges $e \in \mathbf{E}$. Here is our main result (conjectured in Aldous, 2013, sec. 7.4). Given a pair $\left(v^{\prime}, v^{\prime \prime}\right)$, there is a random path $\boldsymbol{\pi}\left(v^{\prime}, v^{\prime \prime}\right)$ that attains the FPP time $X\left(v^{\prime}, v^{\prime \prime}\right)$. Define $\Xi\left(v^{\prime}, v^{\prime \prime}\right):=\max \left\{\xi_{e}: e \in\right.$ $\left.\boldsymbol{\pi}\left(v^{\prime}, v^{\prime \prime}\right)\right\}$ as the maximum edge-traversal time in this minimal-time path. Recall the " $L^{0}$ norm"

$$
\|V\|_{0}:=\inf \{\delta: \mathbb{P}(|V|>\delta) \leq \delta\} .
$$

Theorem 2.2. There exist functions $\psi^{+}$and $\psi_{-}:(0,1] \rightarrow(0, \infty)$ such that $\psi^{+}(\delta) \downarrow$ 0 as $\delta \downarrow 0$, and $\psi_{-}(\delta)>0$ for all $\delta>0$, and such that, for all finite connected edgeweighted graphs and all vertex pairs $\left(v^{\prime}, v^{\prime \prime}\right)$,

$$
\psi_{-}\left(\left\|\frac{\Xi\left(v^{\prime}, v^{\prime \prime}\right)}{\mathbb{E} X\left(v^{\prime}, v^{\prime \prime}\right)}\right\|_{0}\right) \leq \frac{\text { s.d. }\left(X\left(v^{\prime}, v^{\prime \prime}\right)\right)}{\mathbb{E} X\left(v^{\prime}, v^{\prime \prime}\right)} \leq \psi^{+}\left(\left\|\frac{\Xi\left(v^{\prime}, v^{\prime \prime}\right)}{\mathbb{E} X\left(v^{\prime}, v^{\prime \prime}\right)}\right\|_{0}\right) .
$$

In words, $X / \mathbb{E} X$ has small spread if and only if $\Xi / \mathbb{E} X$ is small. Intuition for this result comes from the "almost disconnected" case where the path $\boldsymbol{\pi}\left(v^{\prime}, v^{\prime \prime}\right)$ must contain a specific "bridge" edge $e$ with small $w_{e}$; if $1 / w_{e}$ is not $o(\mathbb{E} X)$ then the contribution to $\mathrm{X}$ from the traversal time $\xi_{e}$ is enough to show that $X$ cannot have weakly concentrated distribution.

The proof of the upper bound starts from our "more general" inequality, Lemma 1.2. See section 3 for an outline.

Theorem 2.2 is unsatisfactory in that the conditions are not directly on the edgeweights $\mathbf{w}=\left(w_{e}\right)$. By analogy with the bounds (Montenegro and Tetali, 2006, sec. 3.2) for Markov chain mixing times in terms of the spectral profile, it seems likely that Proposition 2.1 can be extended to give more widely applicable upper bounds on spread in terms of extremal flow rates $w\left(S, S^{c}\right)=\sum_{v \in S, y \in S^{c}} w_{v y}$. However we do not have any conjecture for two-sided bounds analogous to Theorem 2.2.

A particular case of FPP in our setting - edge-weights $w_{e}$ varying widely over the different edges - is studied in detail in recent work of Chatterjee and Dey (2013). In their model $\mathbf{V}=\mathbb{Z}^{d}$ with $w_{x y}=\|y-x\|^{-\alpha+o(1)}$. Results and conjectures from that paper are consistent with our Theorem 2.2, which says that properties $X(\mathbf{0}, n z) / \mathbb{E} X(\mathbf{0}, n z) \rightarrow_{p} 1$ and $\Xi(\mathbf{0}, n z) / \mathbb{E} X(\mathbf{0}, n z) \rightarrow_{p} 1$ must either both hold or both fail. They identify several qualitatively different regimes. In their linear growth regime $(\alpha>2 d+1)$ they show $\mathbb{E} X(\mathbf{0}, n z) / n$ converges to a nonzero constant and show that both properties hold. In their super-linear growth regime $(\alpha \in(2 d, 2 d+1))$ they show $X(\mathbf{0}, n z)=n^{\alpha-2 d+o(1)}$; here their analysis suggests both properties fail. For $\alpha \in(d, 2 d)$ they show $X(\mathbf{0}, n z)$ grows as a power of $\log n$, and their arguments suggest both properties hold. The qualitative behavior and proof techniques in Chatterjee and Dey (2013) are different in these different regimes, whereas our Theorem 2.2 is a single result covering all regimes, albeit a less explicit result.

2.1. Some preliminaries. (a) We will view the FPP process started at $v^{\prime}$ as a process $\left(Z_{t}\right)$ taking values in the (finite) space of subsets $S \subseteq \mathbf{V}$ of vertices, that is as

$$
Z_{t}:=\left\{v: X\left(v^{\prime}, v\right) \leq t\right\}
$$


The assumption of Exponential distributions implies that $\left(Z_{t}\right)$ is the continuoustime Markov chain with $Z_{0}=\left\{v^{\prime}\right\}$ and transition rates

$$
S \rightarrow S \cup\{y\}: \quad \text { rate } w(S, y):=\sum_{s \in S} w_{s y} \quad(y \notin S) .
$$

So we are in the setting of Lemmas 1.1 and 1.2. Given a target vertex $v^{\prime \prime}$ the FPP time $X\left(v^{\prime}, v^{\prime \prime}\right)$ is a stopping time $T$ of the form (1.1), and the function $h(S)$ at (1.2) is just the function

$$
h(S):=\mathbb{E} \min _{v \in S} X\left(v, v^{\prime \prime}\right) .
$$

For a possible transition $S \rightarrow S \cup\{y\}$, by considering the first time $y$ is reached in the process started from $S$ we have $h(S) \leq \frac{1}{w(S, y)}+h(S \cup\{y\})$ and so

$$
h(S)-h(S \cup\{y\}) \leq \frac{1}{w(S, y)} \leq \frac{1}{w_{*}}
$$

for $w_{*}:=\min \left\{w_{e}: \quad e \in \mathbf{E}\right\}$. Now Lemma 1.1 does indeed imply Proposition 2.1, as stated earlier.

(b) FPP times such as $X\left(v^{\prime}, v^{\prime \prime}\right)$ are examples of distributions $Y>0$ with the submultiplicative property

$$
\mathbb{P}\left(Y>y_{1}+y_{2}\right) \leq \mathbb{P}\left(Y>y_{1}\right) \mathbb{P}\left(Y>y_{2}\right) ; \quad y_{1}, y_{2}>0 .
$$

This holds because, if the percolation process has not reached $v^{\prime \prime}$ before time $y_{1}$, we can restart it at $v^{\prime}$ and use monotonicity. We will need the general bound provided by the following straightforward lemma. See Aldous (2016) for a simple proof with $\gamma(u)=\sqrt{24 u}$.

Lemma 2.3. There exists an increasing function $\gamma(u)>0$ with $\gamma(u) \downarrow 0$ as $u \downarrow 0$ such that, for every submultiplicative $Y$ and every event $A$,

$$
\frac{\mathbb{E} Y \mathbb{1}_{A}}{\mathbb{E} Y} \leq \gamma(\mathbb{P}(A))
$$

Note it follows that

$$
\mathbb{P}(Y \geq y) \geq \gamma^{-1}\left(\frac{\mathbb{E} Y 1_{\{Y \geq y\}}}{\mathbb{E} Y}\right)
$$

for the (increasing) inverse function $\gamma^{-1}(u)$ which also satisfies $\gamma^{-1}(u) \downarrow 0$ as $u \downarrow 0$.

(c) We will need an elementary stochastic calculus lemma.

Lemma 2.4. Let $T_{1}$ be a stopping time with (random) intensity $\eta_{t}$ - that is, $\mathbb{P}(t<$ $\left.T_{1}<t+d t \mid \mathcal{F}_{t}\right)=\eta_{t} d t$ on $\left\{T_{1}>t\right\}$. Let $\zeta$ be another stopping time such that $\eta_{t} \geq c$ on $\{\zeta>t\}$, for constant $c>0$. Then

$$
\mathbb{P}\left(T_{1} \leq \zeta \wedge t_{0}\right) \geq\left(1-e^{-c t_{0}}\right) \mathbb{P}\left(\zeta>t_{0}\right) .
$$

Proof: Applying the optional sampling theorem to the martingale $\exp \left(\int_{0}^{t} \eta_{s} d s\right)$. $\mathbb{1}_{\left\{T_{1}>t\right\}}$ and the stopping time $\zeta \wedge t_{0}$ shows

$$
\mathbb{E}\left[\exp \left(\int_{0}^{\zeta \wedge t_{0}} \eta_{s} d s\right) \cdot \mathbb{1}_{\left\{T_{1}>\zeta \wedge t_{0}\right\}}\right]=1 .
$$

Now

and so

$$
\exp \left(\int_{0}^{\zeta \wedge t_{0}} \eta_{s} d s\right) \geq \exp \left(c\left(\zeta \wedge t_{0}\right)\right) \geq 1+\left(e^{c t_{0}}-1\right) \mathbb{1}_{\left\{\zeta>t_{0}\right\}}
$$

$$
\mathbb{P}\left(T_{1}>\zeta \wedge t_{0}\right)+\left(e^{c t_{0}}-1\right) \mathbb{P}\left(T_{1}>\zeta \wedge t_{0}, \zeta>t_{0}\right) \leq 1
$$


That is,

$$
\begin{aligned}
\mathbb{P}\left(T_{1} \leq \zeta \wedge t_{0}\right) & \geq\left(e^{c t_{0}}-1\right) \mathbb{P}\left(T_{1}>\zeta \wedge t_{0}, \zeta>t_{0}\right) \\
& \geq\left(e^{c t_{0}}-1\right)\left(\mathbb{P}\left(\zeta>t_{0}\right)-\mathbb{P}\left(T_{1} \leq \zeta \wedge t_{0}\right)\right)
\end{aligned}
$$

which rearranges to the stated inequality.

\section{Proof of upper bound in Theorem 2.2}

Fix $v^{\prime}$ and as above view the FPP process started at $v^{\prime}$ as the continuous-time Markov chain $\left(Z_{t}\right)$ at $(2.1)$ on the space of subsets of $\mathbf{V}$. Fix a target $v^{\prime \prime} \neq v^{\prime}$ and in the following write $S$ for an arbitrary subset of vertices containing $v^{\prime}$. Write

$$
h(S):=\mathbb{E} \min _{v \in S} X\left(v, v^{\prime \prime}\right)
$$

for the mean percolation time from $S$ to $v^{\prime \prime}$, so $h(S)=0$ iff $v^{\prime \prime} \in S$. So

$$
T:=X\left(v^{\prime}, v^{\prime \prime}\right)=\inf \left\{t: h\left(Z_{t}\right)=0\right\}
$$

is a stopping time for $\left(Z_{t}\right)$. Note that $t \rightarrow h\left(Z_{t}\right)$ is decreasing and $h(S) \leq \mathbb{E} T$.

Outline of proof. Step 1 is to translate Lemma 1.2 into our FPP setting; this shows it is enough to prove that in all transitions $S \rightarrow S \cup\{y\}$ the decrements $h(S)-h(S \cup\{y\})$ are $o(\mathbb{E} T)$; so suppose not, that is suppose some are $\Omega(\mathbb{E} T)$. Step 2 shows that in some such transitions, the used edge $v y$ will (with non-vanishing probability) have traversal time $\xi_{v y}$ also of order $\Omega(\mathbb{E} T)$. Step 3 shows that some such edges will (with non-vanishing probability) be in the minimal path.

Details of proof. Step 1. Substituting $2 \delta$ for $\delta$, Lemma 1.2 says that for arbitrary $\delta, \varepsilon>0$

$$
\frac{\operatorname{var} T}{(\mathbb{E} T)^{2}} \leq 2 \delta+\varepsilon+\frac{\mathbb{E} \int_{0}^{T} \mathbb{1}_{\left\{q_{\delta}\left(Z_{u}\right) \geq \varepsilon\right\}} d u}{\mathbb{E} T}
$$

where

$$
q_{\delta}(S):=\sum_{y: h(S)-h(S \cup\{y\})>2 \delta \mathbb{E} T} w(S, y)(h(S)-h(S \cup\{y\})) .
$$

Informally, as stated in the outline of proof, this shows it will suffice to prove that in transitions $S \rightarrow S \cup\{y\}$ the decrements $h(S)-h(S \cup\{y\})$ are $o(\mathbb{E} T)$.

Step 2. Now fix $0<u_{1}<u_{2}$.

Lemma 3.1. Condition on $Z_{t_{0}}=S_{0}$. The event during $\left[t_{0}, t_{0}+u_{2}\right]$ the process $\left(Z_{t_{0}+u}\right)$ makes a transition $S \rightarrow$ $S \cup\{y\}$ such that $h(S)-h(S \cup\{y\})>\delta \mathbb{E} T$ and using an edge vy for which $\xi_{v y}>u_{1}$

has probability at least $\left(1-\frac{u_{2}}{\delta \mathbb{E} T}\right)^{+}\left(1-\exp \left(\frac{-\left(u_{2}-u_{1}\right) q_{\delta}\left(S_{0}\right)}{2 \mathbb{E} T}\right)\right)$.

Proof: Define

$$
\zeta=\inf \left\{t>t_{0}: h\left(Z_{t}\right) \leq h\left(S_{0}\right)-\delta \mathbb{E} T\right\}
$$

so that, because $t \rightarrow h\left(Z_{t}\right)$ is decreasing,

$$
h\left(S_{0}\right)-h\left(Z_{t_{0}+u}\right) \leq \delta \mathbb{E} T \text { on }\left\{\zeta>t_{0}+u\right\} .
$$

Define

$$
q^{*}(S):=\sum_{y: h(S)-h(S \cup\{y\})>\delta \mathbb{E} T} w\left(S_{0}, y\right)(h(S)-h(S \cup\{y\})) .
$$


Note this is the definition of $q_{\delta}(S)$ modified by changing the constraint " $>2 \delta \mathbb{E} T$ " to " $>\delta \mathbb{E} T$ " and changing $w(S, y)$ to $w\left(S_{0}, y\right)$. Observe the relation, for $Z_{t_{0}+u} \supseteq S_{0}$,

$$
\begin{aligned}
h\left(Z_{t_{0}+u}\right)-h\left(Z_{t_{0}+u} \cup\{y\}\right) & \geq h\left(Z_{t_{0}+u}\right)-h\left(S_{0} \cup\{y\}\right) \\
& =\left[h\left(S_{0}\right)-h\left(S_{0} \cup\{y\}\right)\right]-\left[h\left(S_{0}\right)-h\left(Z_{t_{0}+u}\right)\right] .
\end{aligned}
$$

For $y$ satisfying the constraint

$$
h\left(S_{0}\right)-h\left(S_{0} \cup\{y\}\right)>2 \delta \mathbb{E} T
$$

in the definition of $q_{\delta}\left(S_{0}\right)$, and on the event $\left\{\zeta>t_{0}+u\right\}$, inequalities $(3.2,3.3)$ show that $y$ satisfies the constraint in the definition of $q^{*}\left(Z_{t_{0}+u}\right)$, and also show that

$$
h\left(Z_{t_{0}+u}\right)-h\left(Z_{t_{0}+u} \cup\{y\}\right) \geq \frac{1}{2}\left[h\left(S_{0}\right)-h\left(S_{0} \cup\{y\}\right)\right],
$$

implying that

$$
q^{*}\left(Z_{t_{0}+u}\right) \geq \frac{1}{2} q_{\delta}\left(S_{0}\right) \text { on }\left\{\zeta>t_{0}+u\right\} .
$$

For $S \supseteq S_{0}$ we have, from the definition of $q^{*}(S)$, a crude bound

$$
q^{*}(S) \leq(\mathbb{E} T) \sum_{y: h(S)-h(S \cup\{y\})>\delta \mathbb{E} T} w\left(S_{0}, y\right)
$$

and applying this to the left side of (3.4) gives

$$
\sum_{y: h\left(Z_{t_{0}+u}\right)-h\left(Z_{t_{0}+u} \cup\{y\}\right)>\delta \mathbb{E} T} w\left(S_{0}, y\right) \geq \frac{q_{\delta}\left(S_{0}\right)}{2 \mathbb{E} T} \text { on }\left\{\zeta>t_{0}+u\right\} .
$$

Over the time interval $\left[t_{0}+u_{1}, t_{0}+u_{2}\right]$ the left side is the intensity of an event which implies the event ( $D$, say) in Lemma 3.1 (in particular, an edge $v y$ is used with $v \in S_{0}=Z_{t_{0}}$, and so its "age" $\xi_{v y}$ must be at least $u_{1}$ ). We are now in the setting of Lemma 2.4, which shows that

$$
\mathbb{P}(D) \geq \mathbb{P}\left(\zeta>t_{0}+u_{2}\right)\left(1-\exp \left(\frac{-\left(u_{2}-u_{1}\right) q\left(S_{0}\right)}{2 \mathbb{E} T}\right)\right)
$$

By the martingale property (1.4) of $h\left(Z_{t}\right)+t$ and Markov's inequality

$$
\mathbb{P}\left(\zeta \leq t_{0}+u_{2}\right)=\mathbb{P}\left(h\left(Z_{t_{0}+u_{2}}\right) \leq h\left(S_{0}\right)-\delta \mathbb{E} T\right) \leq \frac{u_{2}}{\delta \mathbb{E} T}
$$

establishing the bound stated in Lemma 3.1.

\section{Step 3.}

Lemma 3.2. Conditional on the process $\left(Z_{u}\right)$ making at time $t_{0}$ a transition $S \rightarrow$ $S \cup\{y\}$ using an edge vy, the probability that edge vy is in the minimal path $\boldsymbol{\pi}\left(v^{\prime}, v^{\prime \prime}\right)$ is at least $\gamma^{-1}\left(\frac{h(S)-h(S \cup\{y\})}{\mathbb{E} T}\right)$, for the inverse function $\gamma^{-1}(\cdot)>0$ at (2.2).

Proof: Condition as stated. So after the transition we have

$$
T-t_{0}=\min (A, B)
$$

where $B=X\left(y, v^{\prime \prime}\right)$ and $A=\min _{v \in S} X^{*}\left(v, v^{\prime \prime}\right)$ where $X^{*}$ denotes the minimum over paths not using $y$. The probability in question equals $\mathbb{P}(B<A)$; note that $A$ and $B$ are typically dependent, and that $A$ has a continuous distribution.

It is easy to check that the solution to the problem

given the (continuous) distribution of $A>0$, construct a r.v. $B^{\prime}>$ 0 to minimize $\mathbb{P}\left(B^{\prime}<A\right)$ subject to the constraint that $\mathbb{E} A-$ $\mathbb{E} \min \left(A, B^{\prime}\right)$ takes a given value 
is to take $B^{\prime}=A \mathbb{1}_{\left\{A \leq a_{0}\right\}}$ for $a_{0}$ chosen to satisfy the constraint. So in our setting,

$$
\mathbb{P}(B<A) \geq \mathbb{P}\left(A>a_{0}\right)
$$

for $a_{0}$ chosen to satisfy

$$
\mathbb{E} A \mathbb{1}_{\left\{A>a_{0}\right\}}=\mathbb{E} A-\mathbb{E} \min (A, B)=h(S)-h(S \cup\{y\}) .
$$

Because $A$ has the submultiplicative property, Lemma 2.3 shows that

$$
\mathbb{P}\left(A>a_{0}\right) \geq \gamma^{-1}\left(\frac{h(S)-h(S \cup\{y\})}{\mathbb{E} A}\right) .
$$

Because $\gamma^{-1}(\cdot)$ is increasing and $\mathbb{E} A \leq \mathbb{E} T$, we have established the lemma.

Step 4. We now combine the ingredients above. Define $U_{1}=\inf \left\{t \geq 0: q_{\delta}\left(Z_{t}\right) \geq\right.$ $\varepsilon\}$ and inductively

$$
U_{j+1}=\inf \left\{t \geq U_{j}+u_{2}: q_{\delta}\left(Z_{t}\right) \geq \varepsilon\right\} .
$$

Setting $U_{0}=0$, the times $U_{i}$ are such that the sets $\left[U_{i-1}, U_{i}\right] \cap\left\{u: q_{\delta}\left(Z_{u}\right) \geq \varepsilon\right\}$ have Lebesgue measure $u_{2}$. So a sufficient condition to that ensure $U_{j}<\infty$ is the condition that $\left\{u: q_{\delta}\left(Z_{u}\right) \geq \varepsilon\right\} \cap[0, T]$ has Lebesgue measure at least $j u_{2}$, that is

$$
j u_{2} \leq \int_{0}^{T} \mathbb{1}_{\left\{q_{\delta}\left(Z_{u}\right) \geq \varepsilon\right\}} d u \text {. }
$$

Condition on $U_{j}=t_{0}, Z_{U_{j}}=S_{0}$ and apply Lemmas 3.1 and 3.2. We deduce that, with probability at least

$$
\left(1-\frac{u_{2}}{\delta \mathbb{E} T}\right)\left(1-\exp \left(\frac{-\left(u_{2}-u_{1}\right) \varepsilon}{2 \mathbb{E} T}\right)\right) \gamma^{-1}(\delta)
$$

the minimal path $\boldsymbol{\pi}\left(v^{\prime}, v^{\prime \prime}\right)$ contains an edge $v y$ with $\xi_{v y} \geq u_{1}$ and such that $y$ is first reached during $\left(U_{j}, U_{j+1}\right]$. The latter property ensures these edges are distinct as $j$ varies (but note the corresponding $\xi_{v y}$ are dependent). Summing over $j$, applying (3.6) and taking expectation, we find that

$$
N\left(u_{1}\right):=\left|\left\{e \in \boldsymbol{\pi}\left(v^{\prime}, v^{\prime \prime}\right): \xi_{e} \geq u_{1}\right\}\right|
$$

satisfies

$$
\mathbb{E} N\left(u_{1}\right) \geq\left(1-\frac{u_{2}}{\delta \mathbb{E} T}\right)^{+}\left(1-\exp \left(\frac{-\left(u_{2}-u_{1}\right) \varepsilon}{2 \mathbb{E} T}\right)\right) \gamma^{-1}(\delta) \frac{1}{u_{2}} \mathbb{E} \int_{0}^{T} \mathbb{1}_{\left\{q_{\delta}\left(Z_{u}\right) \geq \varepsilon\right\}} d u \text {. }
$$

Writing $\Xi=\Xi\left(v^{\prime}, v^{\prime \prime}\right)$, the event $\left\{N\left(u_{1}\right) \geq 1\right\}$ is the event $\left\{\Xi \geq u_{1}\right\}$. Also $u_{1} N\left(u_{1}\right) \leq T$, so we can write $N\left(u_{1}\right) \leq \frac{T}{u_{1}} \mathbb{1}_{\left\{\Xi \geq u_{1}\right\}}$ and then

$$
\mathbb{E} N\left(u_{1}\right) \leq \frac{\mathbb{E} T}{u_{1}} \frac{\mathbb{E} T 1_{\left\{\Xi \geq u_{1}\right\}}}{\mathbb{E} T} \leq \frac{\mathbb{E} T}{u_{1}} \gamma\left(\mathbb{P}\left(\Xi>u_{1}\right)\right)
$$

the final inequality by Lemma 2.3. Combining with (3.7) and rearranging gives a bound for the final term of (3.1):

$$
\frac{\mathbb{E} \int_{0}^{T} \mathbb{1}_{\left\{q_{\delta}\left(Z_{u}\right) \geq \varepsilon\right\}} d u}{\mathbb{E} T} \leq \frac{u_{2} \gamma\left(\mathbb{P}\left(\Xi>u_{1}\right)\right)}{u_{1}\left(1-\frac{u_{2}}{\delta \mathbb{E} T}\right)+\left(1-\exp \left(\frac{-\left(u_{2}-u_{1}\right) \varepsilon}{2 \mathbb{E} T}\right)\right) \gamma^{-1}(\delta)} .
$$

This becomes a little easier to understand when we set $u_{1}=u \mathbb{E} T$ and $u_{2}=2 u_{1}$; then (3.1) becomes

$$
\frac{\operatorname{var} T}{(\mathbb{E} T)^{2}} \leq 2 \delta+\varepsilon+\frac{2 \gamma\left(\mathbb{P}\left(\frac{\Xi}{\mathbb{E} T}>u\right)\right)}{\left(1-\frac{2 u}{\delta}\right)^{+}\left(1-\exp \left(\frac{-u \varepsilon}{2}\right)\right) \gamma^{-1}(\delta)} .
$$


We could manipulate this into a complicated expression for an upper bound function $\psi^{+}(\cdot)$ in Theorem 2.2. But it is simpler to observe that existence of some upper bound function is equivalent to the following limit assertion: for weighted graphs $\mathbf{w}^{(n)}$ and FPP times $T^{(n)}=X^{(n)}\left(v^{(n) \prime}, v^{(n) \prime \prime}\right)$

$$
\text { if } \frac{\Xi^{(n)}\left(v^{(n) \prime}, v^{(n) \prime \prime}\right)}{\mathbb{E} T^{(n)}} \rightarrow_{p} 0 \text { then } \frac{\operatorname{var} T^{(n)}}{\left(\mathbb{E} T^{(n)}\right)^{2}} \rightarrow 0 .
$$

And this is immediate from (3.8).

\section{Proof of lower bound in Theorem 2.2}

We described this lower bound assertion in Aldous (2013) sec. 7.4 as "intuitively clear (and not hard to prove)". The intuition is that, given a typical realization of the process where $X=X\left(v^{\prime}, v^{\prime \prime}\right)$ has some value $X_{0}$, and some value $\Xi_{0}$ attained by some $\xi_{e}$, there are other realizations for which this $\xi_{e}$ ranges over the interval $\left[0, \Xi_{0}\right]$, for which therefore the value of $X$ ranges over $\left[X_{0}-\Xi_{0}, X_{0}\right]$, so the order of s.d. $(X)$ should be at least the order of $\Xi$. Formalizing this intuition is not quite trivial. The proof below is rather crude - likely there is a more elegant argument giving a better bound.

Fix a pair $\left(v^{\prime}, v^{\prime \prime}\right)$ and write $X=X\left(v^{\prime}, v^{\prime \prime}\right)$ and $\Xi=\Xi\left(v^{\prime}, v^{\prime \prime}\right)$. So $X$ is a function of the traversal times $\left(\xi_{e}\right)$. If we couple $\left(\xi_{e}, e \in \mathbf{E}\right)$ with a copy $\left(\xi_{e}^{\prime}, e \in \mathbf{E}\right)$ of $\left(\xi_{e}\right)$, then by an elementary inequality

$$
\text { var } X \geq \frac{1}{4} \mathbb{E}\left(X^{\prime}-X\right)^{2} .
$$

We will use the following coupling. Fix $0<a<b$ and write $\mathcal{E}(\lambda ; a, b)$ for the distribution of an Exponential $(\lambda)$ random variable conditioned to lie in $[a, b]$. Define $\left(\xi_{e}^{\prime}, \xi_{e}\right)$ to be independent as $e$ varies, with

if $\xi_{e} \notin[a, b]$ then $\xi_{e}^{\prime}=\xi_{e}$

if $\xi_{e} \in[a, b]$ then $\xi_{e}^{\prime}$ is conditionally independent of $\xi_{e}$ with conditional distribution $\mathcal{E}\left(w_{e} ; a, b\right)$.

Consider the set $D_{a b}$ of edges $e$ in the minimal path $\boldsymbol{\pi}=\boldsymbol{\pi}\left(v^{\prime}, v^{\prime \prime}\right)$ for which $\xi_{e} \in[a, b]$. By considering the same path in the coupled copy,

$$
X^{\prime}-X \leq \sum_{e \in \boldsymbol{\pi}}\left(\xi_{e}^{\prime}-\xi_{e}\right)=\sum_{e \in D_{a b}}\left(\xi_{e}^{\prime}-\xi_{e}\right) .
$$

To analyze this expression we will use the following lemma. For $k \geq 1$ and $s>0$ define

$$
F_{k}(s):=\mathbb{E}\left(\max \left(0, s-\sum_{i=1}^{k} U_{i}\right)\right)^{2}
$$

where the $U_{i}$ are independent $\operatorname{Uniform}(0,1)$.

Lemma 4.1. Let $\left(V_{i}, 1 \leq i \leq k\right)$ be independent $\mathcal{E}\left(\lambda_{i} ; a, b\right)$, for arbitrary $\left(\lambda_{i}\right)$. Then for arbitrary $v_{i} \in[a, b]$,

$$
\mathbb{E}\left(\max \left(0, \sum_{i=1}^{k} v_{i}-\sum_{i=1}^{k} V_{i}\right)\right)^{2} \geq(b-a)^{2} F_{k}\left(\sum_{i=1}^{k} \frac{v_{i}-a}{b-a}\right) .
$$

Proof: The distributions $\mathcal{E}\left(\lambda_{i} ; a, b\right)$ are stochastically decreasing with $\lambda_{i}$, so the left side is minimized in the $\lambda_{i} \rightarrow 0$ limit, which is the uniform distribution on $[a, b]$; the limit value is obtained from (4.3) by scaling. 
We can now combine inequalities (4.2) and (4.4) to deduce that, on the event $\left\{\left|D_{a b}\right|=k\right\}$ for $k \geq 1$,

$$
\mathbb{E}\left(\left(X-X^{\prime}\right)^{2} \mid \xi_{e}, e \in \mathbf{E}\right) \geq(b-a)^{2} F_{k}\left(\sum_{e \in D_{a b}} \frac{\xi_{e}-a}{b-a}\right) .
$$

So

$$
\mathbb{E}\left(X-X^{\prime}\right)^{2} \geq(b-a)^{2} \sum_{k \geq 1} \mathbb{E}\left[\mathbb{1}_{\left\{\left|D_{a b}\right|=k\right\}} F_{k}\left(\sum_{e \in D_{a b}} \frac{\xi_{e}-a}{b-a}\right)\right] .
$$

We need to lower bound the right side. The issue is that, if $w_{e}$ is large then $\xi_{e}-a$ may be small. We handle this issue by considering two different values of $a$. Note that the functions $F_{k}(s)$ are decreasing in $k$ and increasing in $s$. Fix $a_{1}<a_{2}<b$, so that for $1 \leq k \leq K$

$$
\begin{aligned}
F_{k}\left(\sum_{e \in D_{a_{1} b}} \frac{\xi_{e}-a_{1}}{b-a_{1}}\right) & \geq F_{K}\left(\sum_{e \in D_{a_{1} b}} \frac{\xi_{e}-a_{1}}{b-a_{1}}\right) \quad(\text { decreasing in } k) \\
& \geq F_{K}\left(\sum_{e \in D_{a_{2} b}} \frac{\xi_{e}-a_{1}}{b-a_{1}}\right) \quad\left(D_{a_{2} b} \subseteq D_{a_{1} b}\right) \\
& \geq F_{K}\left(\left|D_{a_{2} b}\right| \frac{a_{2}-a_{1}}{b-a_{1}}\right) \quad(\text { increasing in } s) \\
& \left.\geq F_{K}\left(\frac{a_{2}-a_{1}}{b-a_{1}}\right) \mathbb{1}_{\left\{\left|D_{a_{2} b}\right| \geq 1\right\}} \quad \text { (increasing in } s\right) .
\end{aligned}
$$

Applying (4.5) with $a=a_{1}$ and restricting the sum to $1 \leq k \leq K$,

$$
\mathbb{E}\left(X-X^{\prime}\right)^{2} \geq\left(b-a_{1}\right)^{2} F_{K}\left(\frac{a_{2}-a_{1}}{b-a_{1}}\right) \mathbb{P}\left(\left|D_{a_{1} b}\right| \leq K,\left|D_{a_{2} b}\right| \geq 1\right) .
$$

Because $a_{1}\left|D_{a_{1} b}\right| \leq X$, Markov's inequality tells us $\mathbb{P}\left(\left|D_{a_{1} b}\right| \geq K\right) \leq \frac{1}{a_{1} K} \mathbb{E} X$. Also the event $\left\{\left|D_{a_{2} b}\right| \geq 1\right\}$ contains the event $\left\{a_{2} \leq \Xi \leq b\right\}$, so

$$
\mathbb{P}\left(\left|D_{a_{1} b}\right| \leq K,\left|D_{a_{2} b}\right| \geq 1\right) \geq \mathbb{P}\left(\Xi \geq a_{2}\right)-\mathbb{P}(\Xi \geq b)-\frac{1}{a_{1} K} \mathbb{E} X .
$$

Because $\Xi \leq X$ we have $\mathbb{P}(\Xi \geq b) \leq \mathbb{E} X / b$, and combining with (4.6) and (4.1) we conclude

$$
\operatorname{var} X \geq \frac{1}{4}\left(b-a_{1}\right)^{2} F_{K}\left(\frac{a_{2}-a_{1}}{b-a_{1}}\right)\left(\mathbb{P}\left(\Xi \geq a_{2}\right)-\left(\frac{1}{b}+\frac{1}{a_{1} K}\right) \mathbb{E} X\right)^{+} .
$$

This holds for arbitrary $0<a_{1}<a_{2}<b$ and $K \geq 1$. We now take $1>\delta>0$ and choose

$$
a_{2}=\delta \mathbb{E} X ; \quad a_{1}=a_{2} / 2 ; \quad b=3 \mathbb{E} X / \delta
$$

and find

$$
\frac{\operatorname{var} X}{(\mathbb{E} X)^{2}} \geq \frac{1}{4}\left(\frac{3}{\delta}-\frac{\delta}{2}\right)^{2} F_{K}\left(\frac{\delta^{2}}{6-\delta^{2}}\right)\left(\mathbb{P}\left(\frac{\Xi}{\mathbb{E} X} \geq \delta\right)-\left(\frac{\delta}{3}+\frac{2}{\delta K}\right)\right)^{+} .
$$

So finally choosing $K=K(\delta) \geq 6 \delta^{-2}$, we see that the lower bound in Theorem 2.2 holds for

$$
\psi_{-}(\delta):=\sqrt{\frac{1}{4}\left(\frac{3}{\delta}-\frac{\delta}{2}\right)^{2} F_{K}\left(\frac{\delta^{2}}{6-\delta^{2}}\right) \frac{\delta}{3}}
$$




\section{Final remarks}

(a). The key point of results such as Proposition 1.4 and Theorem 2.2 is that the bounds do not depend on the size $n$ of the graph - any simple use of the method of bounded differences in these models would give bounds that did depend on $n$.

(b). A somewhat different general approach to proving weak concentration for general coverage processes, assuming IID random subsets, was given in Aldous (1991), and used to prove weak concentration for Markov chain cover times. The latter result does not seem to follow easily from the methods in this paper.

(c). Theorem 2.2 went beyond Proposition 2.1 by using Lemma 1.2 instead of the simpler Lemma 1.1, and so one can imagine analogous improvements of the kind of results in sections $1.2-1.4$.

(d). There is an analog of our FPP result for bond percolation. Let edges $e$ become "open" at the independent random Exponential times $\xi_{e}$ of rates $w_{e}$. Using Lemma 1.2, it is proved in Aldous (2016) that (under minimal assumptions) the time at

which a giant open component starts to emerge is weakly concentrated around its mean.

(e). Presumably some analog of Theorem 2.2 remains true when the Exponential assumption is relaxed to some much weaker "shape of distribution" assumption, but we have not investigated that setting.

\section{Acknowledgements}

I thank Jian Ding for extensive discussions and for dismissing earlier conjectures, Partha Dey for elucidating the connection with Chatterjee and Dey (2013), and an anonymous referee for thoughtful comments.

\section{References}

D. J. Aldous. Probability approximations via the Poisson clumping heuristic, volume 77 of Applied Mathematical Sciences. Springer-Verlag, New York (1989). ISBN 0-387-96899-7. MR969362.

D. J. Aldous. Threshold limits for cover times. J. Theoret. Probab. 4 (1), 197-211 (1991). MR1088401.

D. J. Aldous. Interacting particle systems as stochastic social dynamics. Bernoulli 19 (4), 1122-1149 (2013). MR3102546.

D. J. Aldous. The incipient giant component in bond percolation on general finite weighted graphs. ArXiv Mathematics e-prints (2016). arXiv: 1604.06741.

D. J. Aldous and J. A. Fill. Reversible Markov chains and random walks on graphs (2002). Unfinished monograph, recompiled 2014, available at http://www . stat . berkeley . edu/ aldous/RWG/book.html.

D. J. Aldous and X. Li. A framework for imperfectly observed networks (2016). In preparation.

A. Auffinger, J. Hanson and M. Damron. 50 years of first passage percolation. ArXiv Mathematics e-prints (2015). arXiv: 1511.03262.

I. Benjamini and R. Tessera. First passage percolation on nilpotent Cayley graphs. Electron. J. Probab. 20, no. 99, 20 (2015). MR3399835. 
S. Bhamidi, R. van der Hofstad and G. Hooghiemstra. First passage percolation on random graphs with finite mean degrees. Ann. Appl. Probab. 20 (5), 1907-1965 (2010). MR2724425.

S. Bhamidi, R. van der Hofstad and G. Hooghiemstra. First passage percolation on the Erdős-Rényi random graph. Combin. Probab. Comput. 20 (5), 683-707 (2011). MR2825584.

S. Chatterjee. The universal relation between scaling exponents in first-passage percolation. Ann. of Math. (2) 177 (2), 663-697 (2013). MR3010809.

S. Chatterjee and P. S. Dey. Multiple phase transitions in long-range first-passage percolation on square lattices. ArXiv Mathematics e-prints (2013). arXiv: 1309.5757.

M. Draief and L. Massoulié. Epidemics and rumours in complex networks, volume 369 of London Mathematical Society Lecture Note Series. Cambridge University Press, Cambridge (2010). ISBN 978-0-521-73443-1. MR2582458.

P. Hall. Introduction to the theory of coverage processes. Wiley Series in Probability and Mathematical Statistics: Probability and Mathematical Statistics. John Wiley \& Sons, Inc., New York (1988). ISBN 0-471-85702-5. MR973404.

H. Kesten. On the speed of convergence in first-passage percolation. Ann. Appl. Probab. 3 (2), 296-338 (1993). MR1221154.

H. Kesten. First-passage percolation. In From classical to modern probability, volume 54 of Progr. Probab., pages 93-143. Birkhäuser, Basel (2003). MR2045986.

D. A. Levin, Y. Peres and E. L. Wilmer. Markov chains and mixing times. American Mathematical Society, Providence, RI (2009). ISBN 978-0-8218-4739-8. MR2466937.

C. McDiarmid. On the method of bounded differences. In Surveys in combinatorics, 1989 (Norwich, 1989), volume 141 of London Math. Soc. Lecture Note Ser., pages 148-188. Cambridge Univ. Press, Cambridge (1989). MR1036755.

R. Montenegro and P. Tetali. Mathematical aspects of mixing times in Markov chains. Found. Trends Theor. Comput. Sci. 1 (3), x+121 (2006). MR2341319.

J. Peng. Volatilities analysis of first-passage time and first-return time on a smallworld scale-free network. J. Stat. Mech. Theory Exp. 2016 (3), 033103, 21 (2016). MR3482870.

J. M. Steele. Probability theory and combinatorial optimization, volume 69 of CBMS-NSF Regional Conference Series in Applied Mathematics. Society for Industrial and Applied Mathematics (SIAM), Philadelphia, PA (1997). ISBN 0-89871-380-3. MR1422018. 